\title{
Linfoadenitis criptococócica mesentérica: Una rara causa de abdomen agudo. Caso clínico
}

\author{
OSCAR TAPIA E., MIGUEL VILLASECA H., JUAN CARLOS ARAYA O.
}

\section{Mesenteric cryptococcal lymphadenitis. Report of one case}

Cryptococcosis is an invasive mycotic infection caused by Cryptococcus neoformans, an encapsulated, yeast-like fungus. It is considered an opportunist infection, since it mainly affects immunocompromised subjects. However there are isolated reports of the infection in immunocompetent subjects. Cryptococcal infection of intra-abdominal organs or tissues is extremely rare. We report a 21-year-old HIV positive male that, during the treatment of a meningeal cryptococcosis, presented a clinical picture of an acute abdomen suggesting acute appendicitis. The patient was operated, finding enlarged mesenteric lymph nodes forming conglomerates and a macroscopically normal appendix. The conglomerated lymph nodes and the appendix were excised. The pathological study of the surgical piece revealed an intra-abdominal cryptococcal lymphadenitis and a normal appendix.

(Rev Med Chile 2010; 138: 1535-1538).

Key words: Cryptococcus neoformans; HIV; Mesenteric lymphadenitis.

Departamento de Anatomía
Patológica. Facultad de
Medicina. Universidad de La
Frontera. Temuco, Chile.
Scientific and Technological
Bioresource Nucleus (BIOREN).
Recibido el 2 de marzo de
2010, aceptado el 27 de
septiembre de 2010.
Correspondencia a:
Dr. Oscar Tapia E.
Departamento de Anatomía
Patológica, Facultad de
Medicina, Universidad de La
Frontera. Manuel Montt 112.
Fono: 045-296530. Código
Postal 478-1176. Temuco,
Chile.
E- mail: otescalona@gmail.
com

L a criptococosis corresponde a una infección micótica invasiva causada por un microorganismo levaduriforme encapsulado denominado Cryptococcus neoformans (CpN). Este germen se encuentra ampliamente distribuido en el mundo, siendo sus principales reservorios ambientales el suelo y las heces de animales, preferentemente de aves y murciélagos ${ }^{1-4}$. Es considerado un agente de infecciones oportunistas, afectando principalmente a sujetos inmunocomprometidos tales como pacientes con linfoma, leucemia, VIHSIDA, trasplantados o en tratamiento prolongado con corticoides, sin embargo, existen reportes aislados de afección en sujetos inmunocompetentes ${ }^{1,5-8}$. Este hongo ingresa al organismo mediante la inhalación desde el ambiente, afectando primariamente al tracto respiratorio y produciendo un cuadro clínicamente inespecífico (forma respiratoria). A partir de la localización primaria pulmonar, ésta puede diseminarse a otros órganos o tejidos tales como sistema nervioso central, piel, mucosas y ganglios linfáticos (forma diseminada) $2,3,7,9,10$.

El compromiso de órganos o tejidos intraabdominales es extremadamente raro, más aun el compromiso de ganglios linfáticos a este nivel, pudiendo simular un cuadro de abdomen agudo quirúrgico, donde el diagnóstico es finalmente confirmado mediante el estudio histopatológico del o los ganglios linfáticos comprometidos, representando por otra parte, esta entidad (criptococosis extra-pulmonar) uno de los criterios diagnósticos de VIH-SIDA propuestos por el CDC $C^{3,10-13}$.

\section{Caso clínico}

Hombre de 19 años con antecedentes de VIH diagnosticado hace 6 años, hasta ahora sin trata- 


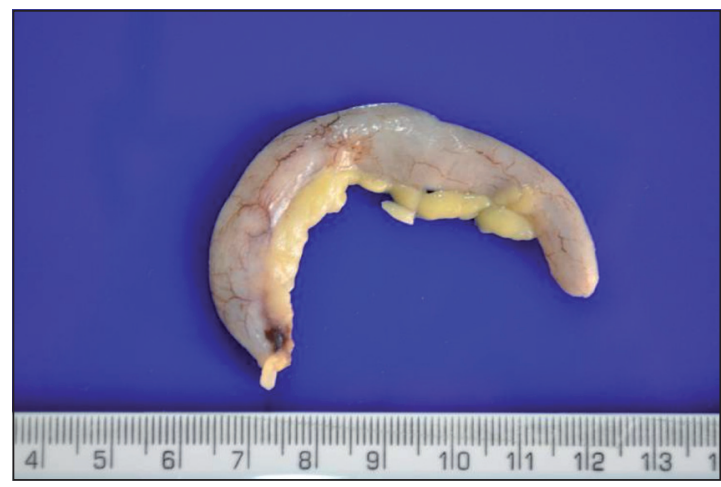

Figura 1. Apéndice cecal de estructura conservada con discreta hiperemia de vasos sanguíneos de la subserosa. miento antiretroviral. Estando hospitalizado por una criptococosis meníngea en tratamiento con anfotericina $\mathrm{B}$, presentó al $8^{\circ}$ día dolor abdominal difuso asociado a fiebre, vómitos y compromiso del estado general. El hemograma mostró 6.470 leucocitos, hematocrito $27,4 \%$, amilasa sanguínea $34 \mathrm{U} / \mathrm{L}$, con pruebas hepáticas y función renal normales. En las 2 horas siguientes evolucionó con mayor dolor abdominal y tendencia a la localización en fosa ilíaca derecha, persistencia de la fiebre hasta $38,9^{\circ} \mathrm{C}$, en ausencia de signos de irritación peritoneal. Con la sospecha clínica de apendicitis aguda se efectuó una laparotomía exploradora, evidenciándose un apéndice cecal de apariencia
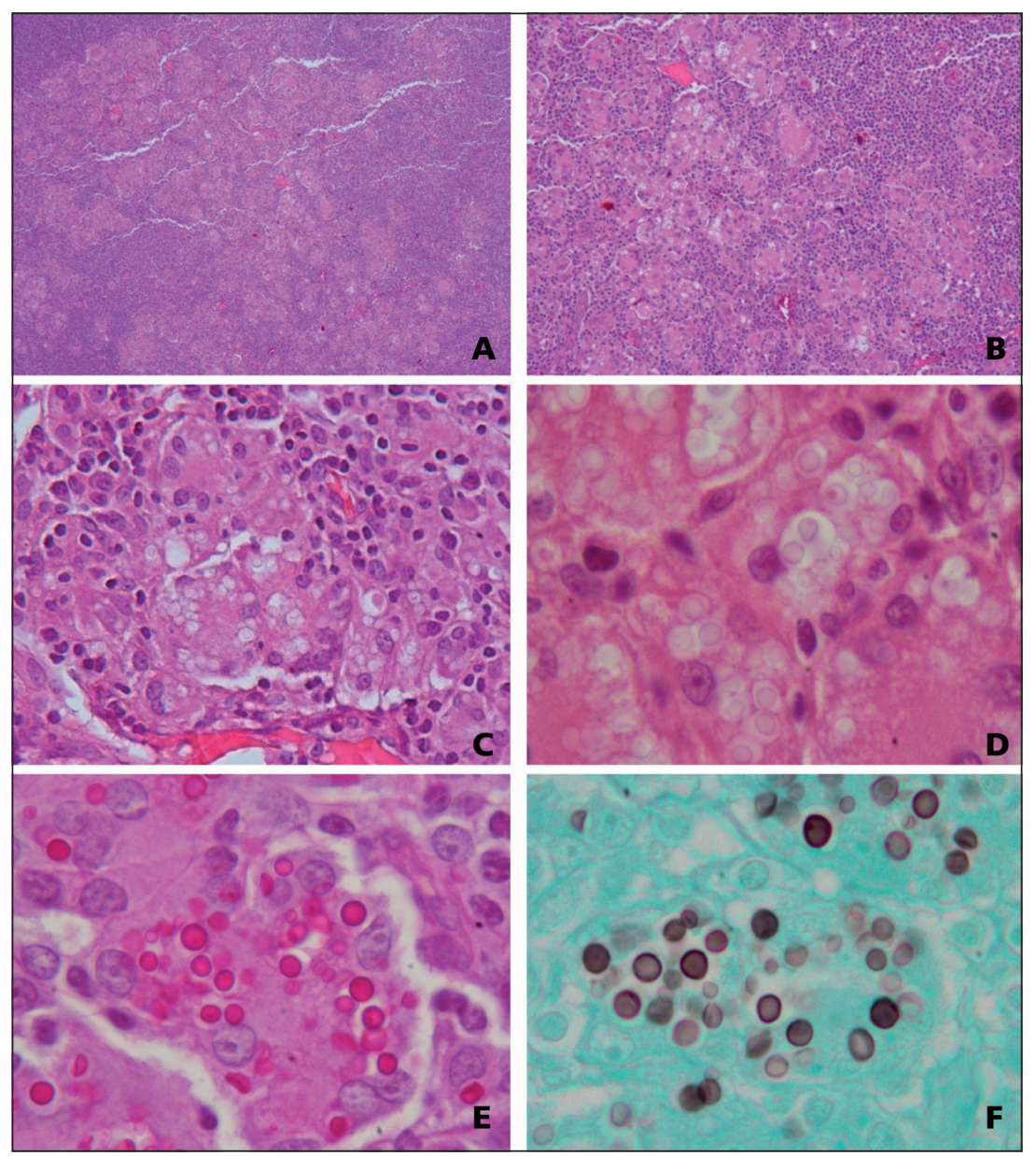

Figura 2. $A(40 x \quad H-E)$, $B(100 x, H-E), C(400 x, H-E)$, D (1000x, H-E), E (1000x, PAS) y $F(1000 x$, Grocott). Se aprecia ganglio linfático con estructura distorsionada por acúmulos de histiocitos y células gigantes multinucleadas ( $A$ y $B$ ), las que presentan en su citoplasma múltiples estructuras levaduriformes refringentes y con cápsula gruesa (C y D), visibles con mayor claridad en tinción de PAS (E) y Grocott (F), compatibles con Criptococo neoformans. 
macroscópica sana y múltiples adenopatías mesentéricas, algunas formando conglomerados de ganglios linfáticos. Se realizó apendicectomía y resección parcial del conglomerado de ganglios linfáticos mesentéricos.

Para estudio histo-patológico se recibió un apéndice cecal de $7,5 \mathrm{~cm}$ de longitud y $0,8 \mathrm{~cm}$ de diámetro con serosa pardo rosada y discreta hiperemia de vasos sanguíneos subserosos (Figura 1) $y$, por separado, un fragmento irregular de tejido fibroadiposo de 1,9 x $1,5 \mathrm{~cm}$ que incluía un conglomerado de estructuras ganglionares linfáticas. El examen microscópico mostró ganglios linfáticos de arquitectura distorsionada por acúmulos de histiocitos y células gigantes multinucleadas, en el interior de las cuales se observan múltiples microorganismos levaduriformes pequeños, de entre $5 \mathrm{y}$ 15 micras, que con tinción de hematoxilina-eosina presentan una cápsula refringente y gruesa, visibles con mayor claridad al realizar tinciones de PAS y Grocott (Figura 2). El examen microscópico del apéndice cecal no evidenció signos inflamatorios. En base a estos hallazgos se informó una linfoadenitis criptococócica mesentérica.

El paciente evolucionó sin complicaciones post-operatorias, completando 15 días de tratamiento antifúngico con anfotericina $\mathrm{B}$ y una respuesta satisfactoria. Al momento del alta se programó iniciar terapia antiretroviral.

\section{Discusión}

La criptococosis es una infección micótica invasiva causada por $\mathrm{CpN}$, el cual se encuentra ampliamente distribuido en el mundo, siendo sus principales reservorios ambientales el suelo $y$ las heces de animales ${ }^{1-4}$. Constituye un agente infeccioso oportunista pues afecta principalmente a sujetos inmunocomprometidos (linfoma, leucemia, VIH-SIDA, trasplantados, etc). El rápido aumento de la incidencia de infección por VIH en el mundo ha generado un aumento de su incidencia y consecuentemente de su relevancia clínicoepidemiológica. Este microorganismo ingresa mediante inhalación al tracto respiratorio llegando hasta los pulmones, estadio que suele ser asintomático o con escasa sintomatología inespecífica que tiende a la resolución espontánea o, en casos aislados y según el compromiso inmunológico del paciente, a la progresión. A partir de la localización primaria pulmonar puede diseminarse por vía hematógena a otros órganos o tejidos tales como sistema nervioso central, piel, mucosas y ganglios linfáticos (forma diseminada). El compromiso extrapulmonar más frecuentemente reportado ocurre en el sistema nervioso central, seguido por la localización cutánea ${ }^{1,2,5-9}$.

La afección de órganos o tejidos intraabdominales es extremadamente rara. La infección criptococócica diseminada, con compromiso de órganos abdominales, presenta dos formas clínicas: una linfonodular, caracterizada por compromiso diseminado de ganglios linfáticos como ocurre en el presente caso, mientras la segunda corresponde a una peritonitis criptococócica generalmente en pacientes en diálisis peritoneal ${ }^{10,14-17}$. A la fecha existen escasos reportes de criptococosis intraabdominal, de las cuales la mayoría corresponden a compromiso peritoneal y minoritariamente a la forma linfonodular ${ }^{3,7,10-12,15-18}$. Nuestro caso representa una forma diseminada de criptococosis con afección tanto meníngea como ganglionar linfática a nivel mesentérico, siendo la presentación clínica de esta infección típicamente de evolución crónica o subaguda. Nuestro paciente tuvo una presentación inusual, con el compromiso mesentérico como un abdomen agudo sugerente de una apendicitis aguda, la que fue descartada por los hallazgos intra-operatorios y el examen anatomo-patológico. Más infrecuente aun es el compromiso aislado de ganglios linfáticos abdominales, tal como reportaron Karagüzel et $\mathrm{al}^{10}$.

Para el diagnóstico de infección por $\mathrm{CpN}$ se requiere de un alto índice de sospecha, debiendo incluir el estudio diagnóstico la toma de muestras tanto para exámenes microbiológicos como histopatológicos con técnicas histoquímicas especiales para la detección de elementos micóticos. En extendidos citológicos y cortes histológicos se observan microorganismos levaduriformes que miden entre 5 y 20 micras, que presentan una cápsula refringente y gruesa; visibles con mayor claridad en cortes histológicos con tinciones histoquímicas especiales, tales como PAS, Grocott, azul alcián y mucicarmín de Best ${ }^{3,7,10}$. En nuestro caso, no se dispuso de material para cultivo, por lo que la biopsia ganglionar constituyó la herramienta diagnóstica.

Si bien la afección de órganos o tejidos abdominales por $\mathrm{CpN}$ es poco frecuente, el progresivo aumento en la incidencia de la infección por VIH 
a nivel mundial y nacional, hacen que hoy el médico clínico deba tener un alto índice de sospecha frente a cuadros clínicos, tanto de presentación clásica como atípica, por agentes oportunistas en pacientes inmunocomprometidos. Del mismo modo el anatomopatólogo debe estar familiarizado con la morfología y cambios histo-patológicos asociados con los distintos microorganismos, y de esta manera efectuar un diagnóstico oportuno y adecuado que permitan la instauración precoz de terapias sistémicas.

\section{Referencias}

1. Bennet JE. Cryptococcosis. In: Braunwald E, Fauci AS, Kasper DL, Hauser SL, Longo DL, James JL, editors, Harrison's Principles of Internal Medicine. 15th ed. New York: McGraw-Hill 2001; 1174-5.

2. Das BP, Panda PL, Mallik RN, Das B. Cryptococcal lymphadenitis and meningitis in human immunodeficiency virus infection: A case report. Indian J Pathol Microbiol 2002; 45: 349-51.

3. Suchitha S, Sheeladevi CS, Sunila R, Manjunath GV. Fine needle aspiration diagnosis of cryptococcal lymphadenitis: A window of opportunity. J Cytol 2008; 25: 147-9.

4. Chakrabarti A. Microbiology of systemic fungal infections. J Postgrad Med 2005; 51 Suppl 1: S16-20.

5. Waters L, Nelson M. Cryptococcal disease and HIV infection. Expert Opin Pharmacother 2005; 6: 2633-44.

6. Sun HY, Chen MY, Hsiao CF, Hsieh SM, Hung CC, Chang SC. Endemic fungal infections caused by Cryptococcus neoformans and Penicillium marneffei in patients infected with human immunodeficiency virus and treated with highly active anti-retroviral therapy. Clin Microbiol Infect 2006; 12: 381-8.

7. Kim SH, Kim SD, Kim HR, Yoon CH, Lee SH, Kim HY et al. Intraabdominal cryptococcal lymphadenitis in a patient with systemic lupus erythematosus. J Korean Med Sci 2005; 20: 1059-61.
8. Chaudhary MW, Sardana K, Kumar P, Dewan V, Anand VK. Disseminated infection with Cryptococcus neoformans var neoformans in an 8 years immunocompetent girl. Indian J Pediatr 2005; 72: 85

9. Lewis JL, Rabinovich S. The wide spectrum of cryptococcal infections. Am J Med 1972; 53: 315-22.

10. Karagüzel G, Kiliçarslan-Akkaya B, Meliko lu M, Karpuzo lu G. Cryptococcal mesenteric lymphadenitis: an unusual cause of acute abdomen. Pediatr Surg Int 2004; 20: 633-5.

11. Lee MY, Chung JH, Shin JH, Hwang TJ, Kim KS, Lee JH et al. Lymphonodular cryptococcosis diagnosed by fine needle aspiration cytology in hyper-IgM syndrome. A case report. Acta Cytol 2001; 45: 241-4.

12. Scalfano FP Jr, Prichard JG, Lamki N, Athey PA, Graves RC. Abdominal cryptococcoma in AIDS: a case report. J Comput Tomogr 1988; 12: 237-9.

13. Schneider E, Whitmore S, Glynn KM, Domínguez K, Mitsch A, McKenna MT, et al. Revised surveillance case definitions for HIV infection among adults, adolescents, and children aged $<18$ months and for HIV infection and AIDS among children aged 18 months to $<13$ years-United States, 2008. MMWR Recomm Rep 2008; 57: 1-12.

14. Aronoff SC. Cryptococcus neoformans. In: Behrman RE, Kliegman RM, Jenson HB, editors, Nelson Textbook of Pediatrics, WB Saunders, Philadelphia, 2000; 935-6.

15. Smith JW, Arnold WC. Cryptococcal peritonitis in patients on peritoneal dialysis. Am J Kidney Dis 1998; 11: 430-3.

16. Yinnon AM, Solages A, Treanor JJ. Cryptococcal peritonitis: report of a case developing during continuous ambulatory peritoneal dialysis and review of the literature. Clin Infect Dis 1993; 17: 736-41.

17. Mansoor GA, Ornt DB. Cryptococcal peritonitis in peritoneal dialysis patients: a case report. Clin Nephrol 1994; 41: 230-2.

18. Crum CP, Feldman PS. Cryptococcal peritonitis complicating a ventriculoperitoneal shunt in unsuspected cryptococcal meningitis. Hum Pathol 1981; 12: 660-3. 